\title{
Clinical and Demographic Profile of Anesthesiologists Using Alcohol and Other Drugs under Treatment in a Pioneering Program in Brazil
}

\author{
Hamer Nastasy Palhares Alves ${ }^{1}$, Denise Leite Vieira ${ }^{2}$, Ronaldo Ramos Laranjeira ${ }^{3}$, Joaquim Edson Vieira ${ }^{4}$, \\ Luiz Antônio Nogueira Martins ${ }^{5}$
}

\begin{abstract}
Summary: Palhares-Alves HN, Vieira DL, Laranjeira R, Vieira JE, Nogueira-Martins LA - Clinical and Demographic Profile of Anesthesiologists Using Alcohol and Other Drugs under Treatment in a Pioneering Program in Brazil.

Background and objectives: Anesthesiologists are the majority in impaired-physician programs that assist physicians who abuse psychoactive substances. The aim of this paper is to show a descriptive study about the clinical and sociodemographic profile of a sample of chemically dependent anesthesiologists treated in a reference program. In addition, the objective is to cite the psychiatric comorbities, the most frequently used drugs and the psychosocial and professional repercussions of substance abuse.
\end{abstract}

Method: A cross-sectional and prospective study was conducted, and a socio-occupational questionnaire and a structured interview were carried out to diagnose mental and psychoactive substance use disorders, according to the International Classification of Diseases (the ICD-10). The questionnaire and the structured interview were carried out by two skilled researchers.

Results: Fifty-seven anesthesiologists were interviewed. Most of them were male (77.2\%), and the mean age was 36.1 years (SD = 8.5\%). A high prevalence of abuse of opioid (59.6\%), benzodiazepine (3.1\%) and alcohol (35.1\%) was observed. Opioid users sought treatment earlier than other substance users and usually they were under pressure from their colleagues and the Regional Council of Medicine. The incidence of drug abuse for self-medication was high in this subgroup.

Conclusions: Anesthesiologists may present a different profile concerning the risks of opioid use. Opioid abuse usually begins during medical residency or during the first years of clinical practice, which supports the hypothesis that addiction to opioids is an occupational issue among anesthesiologists.

Keywords: Anesthesiology; Occupational Diseases; Opioid-Related Disorders; Mental Health.

Financial Support: This study was funded by the National Council for Scientific and Technological Development (CNPq) - N 141366/2003-6 and received strategic support from São Paulo State Regional Council of Medicine (CREMESP).

\section{INTRODUCTION}

Drug use is one of the main causes of the loss of work capacity and early retirement ${ }^{1}$. Psychoactive substance abuse among physicians is as prevalent as among the general population ${ }^{2-4}$.

Anesthesiologists are usually the focus of research related to chemical dependence among physicians ${ }^{4-6}$. In a clinical sample of 198 Brazilian substance-addicted physicians, al-

Received from the Drug and Alcohol Research Unit, Department of Psychiatry, Escola Paulista de Medicina - Universidade Federal de São Paulo, Brazil.

1. PhD, Universidade Federal de São Paulo (UNIFESP); Researcher, Drug and Alcohol Research Unit (UNIAD/INPAD)

2. PhD, UNIFESP; Researcher, UNIAD

3. Full Professor, Department of Psychiatry, UNIFESP

4. Professor, Faculdade de Medicina da Universidade de São Paulo (FMUSP); Researcher,

Department of Anesthesiology, FM-USP

5. Associate Professor, Department of Psychiatry, UNIFESP

Submitted on May 20, 2011.

Approved on August 3, 2011.

Correspondence to:

Dr. Hamer Nastasy Palhares-Alves

Rua Borges Lagoa, 564 cj 132

04038002 - São Paulo, SP, Brasil

Email: hamerpalhares@yahoo.com.br though anesthesiologists represent approximately 3\% of physicians, they formed $12.5 \%$ of those under treatment for substance-abuse 7 . Additionally, compared to other physicians, mortality risks related to suicide and chemical dependence is higher among anesthesiologists ${ }^{8}$.

Opioid abuse among anesthesiologists has been widely reported in the medical literature $4,5,9,10$. A study reported that the incidence of opioid abuse among medical residents is $1.6 \%$. Residents are particularly vulnerable to experimentation with drugs ${ }^{11}$.

Physicians, especially anesthesiologists, are under constant pressure related to their jobs, which include an insalubrious environment, emergency-related stress, work overload, and night shift. In addition, there are other two particularly relevant issues: the easy access to highly addictive substances and the habit of self-medicating to relieve insomnia, anxiety and physical pain ${ }^{4,5}$.

This study was aimed at describing the sociodemographic and occupational profile of a sample of anesthesiologists treated in a reference program, reporting the reasons that led physicians to seek treatment and the main psychiatric co- 
morbities, studying the association among various substance abuse patterns, and at discussing the hypothesis of the influence of occupational issues on chemical dependence among anesthesiologists.

\section{METHOD}

This descriptive, cross-sectional and retrospective study was conducted at the Drug and Alcohol Research Unit of the Department of Psychiatry of the Escola Paulista de Medicina, Universidade Federal de São Paulo (Paulista School of Medicine).

The present study is part of the project called "Cuidando de médicos com dependência química: perfil clínico e demográfico de uma amostra de médicos em tratamento ambulatorial" ("Treating Chemical-dependent Physicians: the clinical and demographic profile of physicians treated in outpatient settings") and was approved by the Research Ethics Committee of the Federal University of São Paulo (№ 1230/03).

\section{Sample group}

All anesthesiologists who sought treatment for noxious substance use or substance dependence and who agreed to participate in the study by signing the informed consent form between 2002 and $2009(N=57)$ were included in the sample.

These anesthesiologists sought treatment by means of a specific program for the treatment of physicians. This program provides treatment and orientation and is aimed at guaranteeing quick access to treatment, as well as protecting the public and the physician himself. This program is subsidized by the São Paulo State Regional Council of Medicine (CREMESP).

\section{Procedures}

Two interviewers - psychiatrists who are experts in treating alcohol and other drug dependence - were trained in order to achieve a concordance level of $85 \%$ in 14 training interviews, before data collection. After training, each of the interviewers conducted semi-structured interviews, lasting about 90 minutes, to register sociodemographic and occupational data, patterns of substance use, and psychiatric comorbities. The interviews were carried out when the patient entered treatment, i.e., at the first or second medical appointment. The interviewers established the psychiatric diagnoses based on the Symptom Checklist, according to the ICD-10 criteria 12 .

Correlated variables such as unemployment, marriage conflicts, car accidents, hospitalization for mental illness, professional issues, problems with the Regional Council of Medicine and self-medication were assessed by means of yes/no answers.

\section{Statistical analysis}

Descriptive analyses were conducted in order to describe the sociodemographic profiles of the sample. The categorical variables were described by using simple frequencies. For the numeric variables, the median $(\mathrm{M})$ and the standard deviation (SD) were calculated. The chi-square test $\left(\chi^{2}\right)$ was used to verify associations among variables using a significance level of $5 \%$.

\section{RESULTS}

Fifty-seven anesthesiologists, 44 male (77.2\%) and 13 (22.8\%) female, were evaluated. The mean age of physicians seeking treatment was 36.1 years $(S D=8.5)$.

Most of physicians reported only drug abuse (37 cases or $64.9 \%$ ). A second group reported only alcohol abuse (10 cases or $17.5 \%)$, and another group reported alcohol and other drug abuse (10 cases or $17.5 \%$ ).

Opioid abuse was reported by 34 anesthesiologists (59.6\%), and opioid was the most common drug used by the sample. Among opioid abusers, $88 \%$ showed an intense consumption pattern, which can be linked to dependence (Tables I and II).

Anesthesiologists abusing opioids sought treatment earlier, usually in the first year of the substance dependence $(p=0.048)$.

As for the sequence of substances used in the beginning of drug consumption, one third of the sample (19 cases) developed drug dependence by using opioids, while $16(28.1 \%)$ started with alcohol abuse and $10(17.5 \%)$ with benzodiazepine abuse. Finally, $12(21.0 \%)$ developed dependence with illicit drugs, such as marijuana and cocaine.

Those initiating alcohol abuse took longer to seek treatment $(p=0.037)$. Those using illicit drugs developed dependence on a higher number of substances $(p<0.001)$.

Table I - Frequency of Noxious Use and Dependence among 57 Anesthesiologists*

\begin{tabular}{llll}
\hline Drug of Abuse & Total n (\%) & $\begin{array}{l}\text { Noxious Use } \\
\mathrm{n}(\%)\end{array}$ & $\begin{array}{l}\text { Dependence }^{\star \star \star} \\
\mathrm{n}(\%)\end{array}$ \\
\hline Alcohol & $20(35.1 \%)$ & $7(12.3 \%)$ & $13(22.8 \%)$ \\
Benzodiazepine & $20(35.1 \%)$ & $3(5.2 \%)$ & $17(29.8 \%)$ \\
Opioids & $34(59.6 \%)$ & $4(7.0 \%)$ & $30(52.6 \%)$ \\
Cocaine/Crack & $3(5.2 \%)$ & $3(5.2 \%)$ & $0(0 \%)$ \\
Marijuana & $6(10.5 \%)$ & $4(7.0 \%)$ & $2(3.5 \%)$ \\
Amphetamines & $6(10.5 \%)$ & $2(3.5 \%)$ & $4(7.0 \%)$ \\
Inhalants & $1(1.8 \%)$ & $1(1.8 \%)$ & $0(0 \%)$ \\
\hline
\end{tabular}

${ }^{*}$ The sum can be higher than $100 \%$ because some physicians used more than one substance.

${ }^{\star *}$ Noxious use can be characterized as a problematic substance use behavior, with recurrent psychosocial repercussions that, however, are not considered to be substance dependence.

${ }^{* * *}$ Dependence syndrome occurs when three or more of the following criteria are identified to have been present in the previous year: 1) Strong desire or compulsion to take the substance; 2) Difficulties in controlling substance-taking behavior in terms of its onset, termination, or levels of use; 3) A physiological withdrawal state when substance use has ceased or has been reduced; 4) Tolerance; 5) Progressive neglect of alternative pleasures or interests because of psychoactive substance use; 6) Persisting with substance use despite clear evidence of overtly harmful physical and psychosocial-related problems. 
Table II - Comparison between Anesthesiologists Using Opioids and Anesthesiologists Using Other Psychoactive Substances

\begin{tabular}{|c|c|c|c|}
\hline Variables & Opioid Users $(n=34)$ & Opioid Non-users $(n=23)$ & $\mathrm{p}$ value \\
\hline \multicolumn{4}{|l|}{ Time gap until seeking for treatment } \\
\hline Between 1 and 5 years & $17(50.0 \%)$ & $10(43.5 \%)$ & \\
\hline Between 6 and 15 years & $4(11.8 \%)$ & $9(39.2 \%)$ & \\
\hline More than 15 years & $1(2.9 \%)$ & $1(4.3 \%)$ & \\
\hline Under family pressure & $4(11.8 \%)$ & $10(43.5 \%)$ & \\
\hline Under Colleague or the Regional Council of Medicine Pressure ${ }^{\star *}$ & $15(44.1 \%)$ & $5(21.7 \%)$ & \\
\hline N\# Illicit Drugs Used & 0.94 & 1.39 & $\mathrm{NS}^{*}$ \\
\hline Mean Age & $36.50(\mathrm{SD}=7,68)$ & $41.43(\mathrm{SD}=9,75)$ & 0.037 \\
\hline Professional Issues & $32(94.1 \%)$ & $18(78.3 \%)$ & NS \\
\hline Legal-Ethical Issues & $11(32.4 \%)$ & $3(13.0 \%)$ & NS \\
\hline Redirection to another Medical Specialty & $4(11.8 \%)$ & $1(4.3 \%)$ & NS \\
\hline Marriage Conflicts & $16(47.1 \%)$ & $14(60.9 \%)$ & NS \\
\hline Car Accident & $5(14.7 \%)$ & $7(30.4 \%)$ & NS \\
\hline Psychiatric Comorbidity & $15(44.1 \%)$ & 9 (39.1\%) & NS \\
\hline Hospitalization & $8(23.5 \%)$ & $9(39.1 \%)$ & NS \\
\hline
\end{tabular}

*NS: Non-significant; **Regional Council of Medicine.

The incidence of self-medication was higher among opioid users $(89.5 \%)$ or other drug users $(81.8 \%)$, compared to $50 \%$ of the group of alcohol users $(p=0.021)$.

\section{DISCUSSION}

Studies about physicians and alcohol and other drug addiction can be conducted in three modus operandi ${ }^{13}$ : by means of epidemiological studies, analysis of the cases submitted to disciplinary procedures and the analysis of the people attending specific clinical programs. Substance addiction among physicians is a difficult issue, as there is a strong tendency on the part of physicians to deny or minimize the problem for fear of the consequences related to their jobs or professional status. In the present paper we studied a convenience sample consisting of anesthesiologists seeking treatment due to issues related to noxious use/dependence on alcohol and other drugs in a specific program for treating physicians.

In this sample the use of alcohol and drugs has led to several consequences, such as professional issues (87.7\%), marriage conflicts $(52.6 \%)$, hospitalization for mental illness $(29.1 \%)$, car accidents $(21.1 \%)$ and unemployment in the previous year (17.5\%). Problems with the Regional Council of Medicine were also frequent (24.6\%).
Redirection to another medical specialty, which occurred in five cases $(8.8 \%)$, requires a thorough investigation. It is possibly a necessary measure taken to protect both the physician and the public from the noxious dependence consequences.

In this study we observed significant differences among opioid users and non-users. Opioid users were younger $(p=0.037)$ and sought treatment earlier than the non-users $(p=0.048)$. Additionally, initiation to substance abuse coincided with the beginning of medical residency and the period immediately following residency. Residents seem to be more vulnerable to opioid dependence ${ }^{11}$.

The incidence of self-medication was also greater among opioid users $(91.2 \%)$, compared to $52.5 \%$ of opioid non-users $(p=0.001)$. Therefore, avoiding self-medication can be one of the prevention measures against drug dependence among anesthesiologists.

Opioid users stated different reasons from other abusers for entering treatment. They sought treatment especially because they were under pressure from their colleagues or from the Regional Council of Medicine; family pressure was less common $(p=0.013)$.

Professional problems being more relevant than family issues among opioid-dependent physicians may be due to the fact that substance abuse or diversion occurs in the workplace. It is therefore necessary to instruct physicians so 
that they can be able to detect and manage the opioid-related problems, considering that the family is not always prepared to identify the problem.

We could identify three different subgroups according to the drug initially used. The first group (18 cases or $31.6 \%$ ) started using drugs earlier and abused a greater number of drugs. The second group (10 cases or $17.5 \%$ ) consisted exclusively of alcohol abusers. They sought treatment later and there was also a great time gap between the moment of problem detection and the search for therapeutic support. The third group (29 cases or $50.9 \%$ ) consisted of individuals who did not have any history of substance abuse or dependence until they suddenly started to use opioids or benzodiazepine, usually administered intravenously.

There is a remarkable difference between the profile of the opioid-dependent anesthesiologists shown in this study and the opioid users in the general population ${ }^{14}$, as psychiatric comorbities were less frequent ( $44.1 \%$ vs. $65.2 \%$, respectively), as well as the personality disorders ( $10.5 \%$ vs. $36.7 \%$, respectively). In addition, problems concerning alcohol use were less common ( $23.5 \%$ vs. $65.9 \%$, respectively). Data suggests that, besides personality disorders and psychiatric comorbities, other specific factors may have influenced the development of opioid dependence among anesthesiologists of the sample. Therefore, these findings suggest that the analysis of the psychiatric or personality psychopathology may not be enough to detect individuals more vulnerable to opioid addiction. That is why universal and extensive prevention is recommended to all anesthesiologists.

The results reported here draw attention to occupational factors specific to the anesthesiology specialty, such as insalubrious environments, easy access to highly addictive substances, great expectations to solve problems using medicament ("pharmacological optimism") ${ }^{15}$, lack of any taboo against needles and injections, self-medication and environmental exposure to aerosolized particles of drugs that may produce neurobiological sensitization and make experimentation more likely ${ }^{16}$. There are evidences that fentanyl and propofol are present in the operating room air ${ }^{17}$.

These facts highlight the necessity of acknowledging that chemical dependence among anesthesiologists is probably associated to occupational issues. This paradigm shift would produce considerable change in how to deal with these patients. The attention given by other physicians, the guarantee of labor rights and a more empathetic approach can lead to a more effective and early treatment. Impaired physicians may not seek treatment for fear of professional consequences. Therefore, all physicians should be responsible for actively helping their colleagues to seek the appropriate treatment.

\section{Limitations}

As we used a convenience sample, it is difficult to expand the findings shown in this study to the anesthesiologists in need of medical treatment due to substance abuse. Therefore, data shown in this research is not a representative sample of the population of substance-dependent anesthesiologists. But, as substance-dependent physicians are a "hidden" class to which it is difficult to approach, our data can help understand the problem.

\section{ACKNOWLEDGEMENTS}

We would like to thank the strategic and financial support from the São Paulo State Regional Council of Medicine (CREMESP). 


\section{REFERÊNCIAS/REFERENCES}

1. Murray CJ, Lopez AD - Global mortality, disability, and the contribution of risk factors: Global Burden of Disease Study. Lancet, 1997;349:1436-1442.

2. Brewster JM - Prevalence of alcohol and other drug problems among physicians, JAMA, 1986;255:1913-1920.

3. Newbury-Birch D, Walshaw D, Kamali F - Drink and drugs: from medical students to doctors. Drug Alcohol Depend, 2001;64:265-270.

4. Baldisseri MR - Impaired healthcare professional. Crit Care Med, 2007;35:S106-116.

5. Spiegelman WG, Saunders L, Mazze RI - Addiction and anesthesiology, Anesthesiology, 1984;60:335-341.

6. Palhares-Alves HN, Laranjeira RR, Nogueira-Martins LA - A pioneering experience in Brazil: the creation of a support network for alcohol and drug dependent physicians. A preliminary report. Rev Bras Psiquiatr, 2007;29(3):258-261. 
7. Alves HN, Surjan JC, Nogueira-Martins LA et al. - Perfil clínico e demográfico de médicos com dependência química. Rev Assoc Med Bras, 2005;51(3):139-143.

8. Alexander BH, Checkoway $\mathrm{H}$, Nagahama $\mathrm{SI}$ et al. - Cause-specific mortality risks of anaesthesiologists. Anesthesiology, 2000;93:922930.

9. Talbott GD, Gallegos KV, Wilson PO, et al. - The medical association of georgias impaired physicians program. Review of the first 1000 physicians: analysis of specialty. JAMA, 1987;257:2927-2930.

10. Kintz $P$, Villain $M$, Dumestre $V$ et al. - Evidence of addiction by anesthesiologists as documented by hair analysis. Forensic Sci Int, 2005;153(1):81-84.

11. Booth JV, Grossman D, Moore J et al. - Substance abuse among physicians: a survey of academic anesthesiology programs. Anesth Analg, 2002;95:1024-1030.

12. Janca A, Hiller W - ICD-10 checklists -a tool for clinicians use of the ICD-10 classification of mental and behavioral disorders. Compr Psychiatry, 1996;37:180-187.

13. Katsavdakis KA, Gabbard GO, Athey GI - Profiles of impaired health professionals. Bull Menninger Clin, 2004;68:60-72.

14. Regier DA, Farmer ME, Rae DS et al - Comorbidity of mental disorders with alcohol and other drug abuse. Results from the Epidemiologic Catchment Area (ECA) Study. JAMA, 1990;264:2511-2518.

15. Kenna GA; Wood MD - In search of pharmacological optimism: Investigating beliefs about effects of drugs: A pilot study. Addict Res Theor, 2008;16(4):383-399.

16. Gold MS, Melker RJ, Dennis DM et al. - Fentanyl abuse and dependence: further evidence for second hand exposure hypothesis. J Addict Dis, 2006;25:15-21.

17. Merlo LJ, Goldberger BA, Kolodner D et al. - Fentanyl and propofol exposure in the operating room: sensitization hypotheses and further data. J Addict Dis, 2008;27(3):67-76. 